\title{
Independent Hox-Cluster Duplications in Lampreys
}

\author{
Claudia Fried ${ }^{\ddagger}, \dagger$, Sonja J. Prohaska ${ }^{\ddagger}, \dagger$ \\ AND PETER F. STADLER ${ }^{\ddagger} \dagger$ \\ ${ }^{\ddagger}$ Bioinformatik, Institut für Informatik, \\ Universität Leipzig, Kreuzstraße 7b, D-04103 Leipzig, Germany \\ †Institut für Theoretische Chemie und Molekulare Strukturbiologie Universität \\ Wien, \\ Währingerstraße 17, A-1090 Wien, Austria \\ *Address for correspondence: \\ Peter F. Stadler, Bioinformatik, Institut für Informatik, Universität Leipzig, \\ Kreuzstraße 7b, D-04103 Leipzig, Germany. Phone: ++49 341149 5120; Fax: \\ ++49 341149 5119; Email: peter.stadler@bioinf.uni-leipzig.de.
}

\begin{abstract}
.
The analysis of the publicly available Hox gene sequences from the sea lamprey Petromyzon marinus provides evidence that the Hox clusters in lampreys and other vertebrate species arose from independent duplications. In particular, our analysis supports the hypothesis that the last comman ancestor of agnathans and gnathostomes had only a single Hox cluster which was subsequently duplicated independently in the two lineages.
\end{abstract}

Keywords. Hox clusters, lamprey, phylogenetic footprints 


\section{Introduction}

Hox genes code for homeodomain containing transcription factors which are homologous to the genes in the Drosophila homeotic gene clusters (McGinnis and Krumlauf, 1992; Schubert et al., 1993). There is good evidence that the common ancestor of sharks, bony fish, and tetrapods, had four clusters homologous to the mammalian ones (Holland and Garcia-Fernandez, 1996; Prohaska et al., 2003b). An additional duplication event in the teleost lineage increased the number of distinct clusters to at least 7, e.g. in zebrafish (Amores et al., 1998; Stellwag, 1999).

The agnathan vertebrates, lampreys (Hyperoartia) and hagfishes (Hyperotreti), as the most primitive extant true vertebrates, occupy a phylogenetically intermediate position between the cephalochordates, such as amphioxus, with a single Hox cluster (Garcia-Fernández and Holland, 1994) and the gnathostomes with four or more clusters. PCR surveys (Pendleton et al., 1993; Sharman and W., 1998) and recent genomic mapping data (Force et al., 2002; Irvine et al., 2002) indicate that lampreys have at least three and possibly four Hox clusters, Fig. 1.

Despite recent efforts, the evolutionary history of the lamprey Hox genes and their relationship with the quadruplicate mammalian Hox clusters is far from being resolved. Irvine et al. (2002) conclude that they have "insufficient data to determine with confidence the identities and evolutionary histories of the lamprey Hox clusters." Amores et al. (1998) argue for a two-step duplication scenario, with a duplication of both ancestral agnathan clusters, possibly simultaneously by genome duplication, to produce the four cluster ancestral gnathostome arrangement. Force et al. (2002) report that "in general, the lamprey Hox genes do not appear to be orthologues of specific Hox genes in gnathostomes" and conclude that the most likely scenario is one genome duplication in the vertebrate ancestor producing a $\operatorname{Hox} A B$ and a $\operatorname{Hox} C D$ cluster with subsequent divergence of the agnathan and gnathostome lineages and independent subsequent duplications in each linage. Ample evidence from other gene families (Escriva et al., 2002), including Dlx (Neidert et al., 2001) and Otx (Germot et al., 2001) confirms at least one independent duplication in the agnathan and

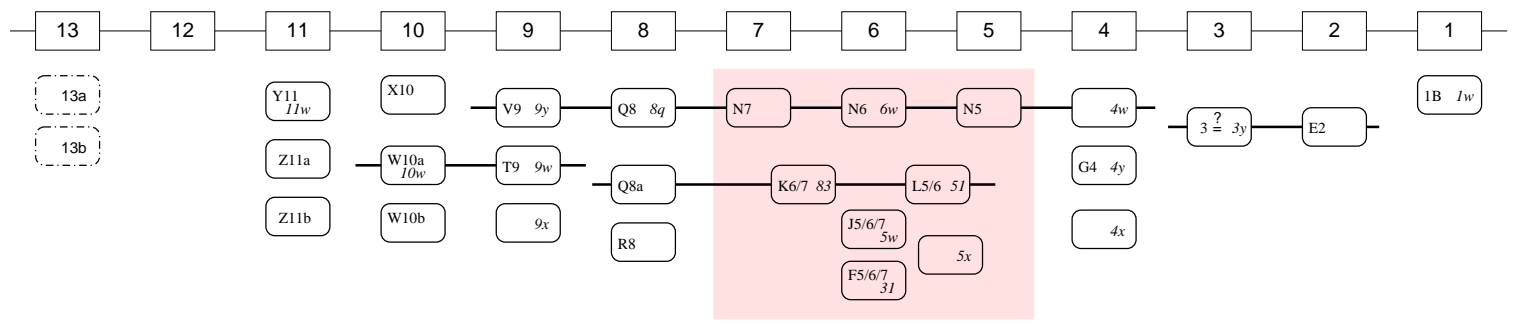

Figure 1. Petromyzon marinus Hox clusters. Summarized from (Force et al., 2002), Fig.1, and (Irvine et al., 2002), Fig.1 and Table 1. Hox13 genes identfied in the PCR survey (Force et al., 2002) but for which no cDNA or cosmid was reported in (Force et al., 2002; Irvine et al., 2002) are indicated by dashed boxes. The corresponding sequences are not available. Physical linkage is indicated by a line. The sequences of paralog groups 5, 6, and 7 are insufficient to resolve their mutual relationships, and are therefore excluded from further analysis. 
the gnathostome lineages. In this letter we report on a re-evaluation of the publicly available lamprey Hox sequences.

\section{Materials and Methods}

The available lamprey Hox sequences are compiled (together with their accession numbers) in Table 1 in the Appendix. Only short sequences of the homeobox region are available in almost all cases. In contrast to the previous studies we use the nucleic acid sequences rather than the sequences of the Hox proteins because of the weak phylogenetic signals in the short and highly conserved amino acid sequences. The sequence from the PCR survey of Lampetra planeri (Sharman and W., 1998) are much shorter (82nt) than the Petromyzon marinus sequence reported by Pendleton et al. (1993) (180nt) and Irvine et al. (2002) (240nt). In almost all cases it was possible to identify the homology between the Lampetra planeri sequences and their Petromyzon marinus counterparts, see Table 1. We therefore use the data from Irvine et al. (2002) where possible.

Canonical split decomposition (Bandelt and Dress, 1992), as implemented in the splitstree package (version 3.1) by Huson (1998), is used for the reconstruction of the phylogeny. The split-based methods are particularly suitable for our purposes because they are known to be very conservative in that they tend to produce multifurcations rather than poorly supported edges (Semple and Steel, 2003). For comparison we compute exact maximum parsimony trees using the program dnapenny which is part of the phylip package (Felsenstein, 1989). We use a variety of Hox genes from mammals (Homo sapiens and Rattus norvegicus), shark (Heterodontus francisci), coelacanth (Latimeria menadoensis), and amphioxus (Branchiostoma floridae) for phylogeny reconstruction. All sequences were downloaded from genbank. Alignments were constructed using dialign (Morgenstern, 1999). Since split-based methods tend to lose resolution with increasing number of taxa we use different combinations of lamprey and sequences from other taxa instead of using all sequences together.

An independent line of evidence is derived from the analysis of conserved non-coding DNA. The 30kb PAC clone Pm18 containing the HoxW10a region of Petromyzon marinus was sequenced by Irvine et al. (2002), accession number AF464190. Here we use the tracker program (Prohaska et al., 2003a) to search for phylogenetic footprints in the non-coding parts of this sequence by comparing it with the corresponding regions of the publicly available sequences of human, fugu (Takifugu rubripes, sequences obtained from the JGI database ${ }^{1}$, release 3.0), and shark Hox clusters. In the case of the $H_{0 x B}$ clusters, which lack Hox-10, Hox-11 and Hox-12 gene, we use the complete inter-genic region from Hox-13 to Hox-9 for the tracker run. The output is then restricted to the region between the first and the last footprint that the lamprey sequence shares with another cluster to account for the fact that Pm18 does not span the entire range to the neighboring genes. 


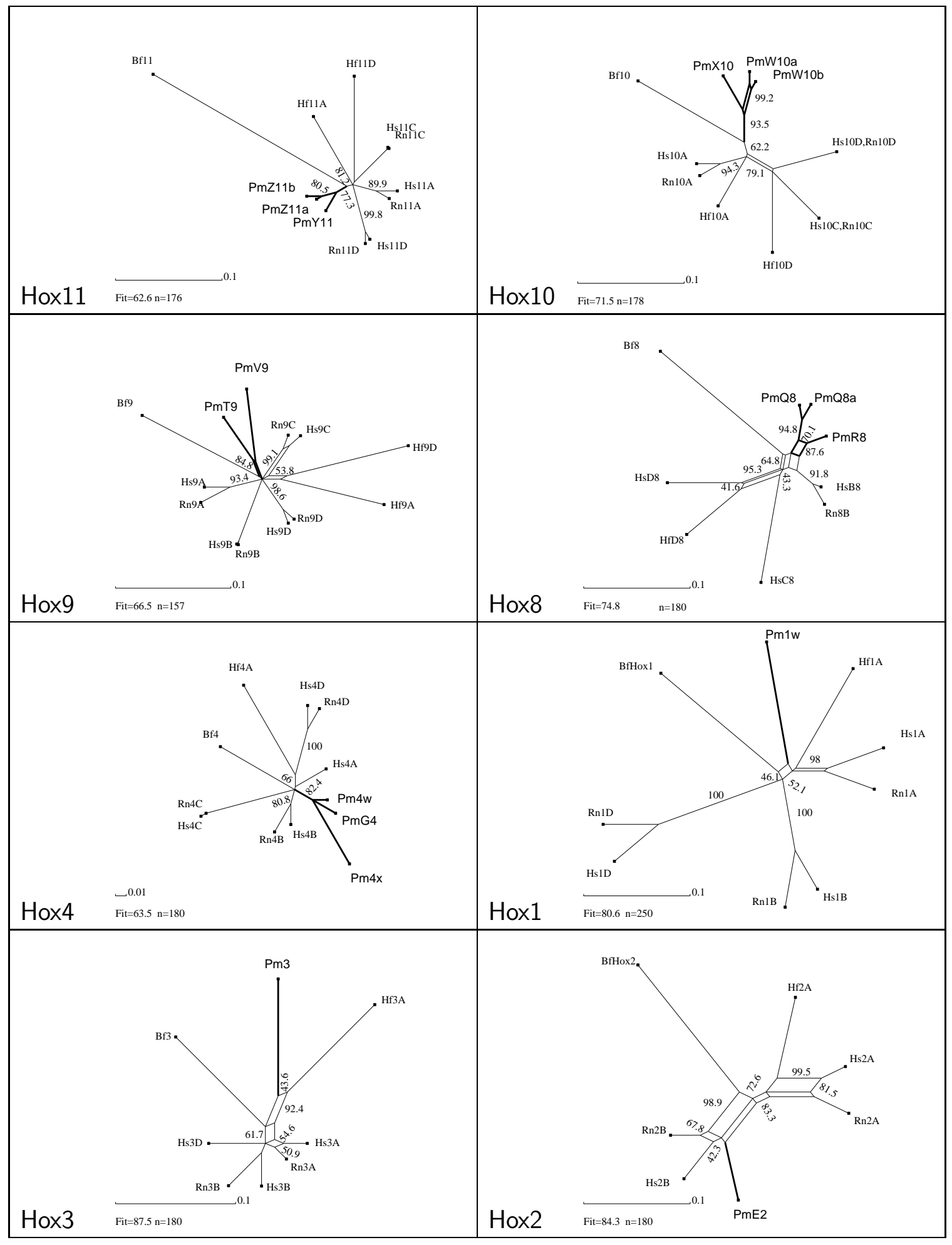

Figure 2. Buneman graphs of the homeobox sequences for paralog groups 1, 2, 3, 4, 8, 9, 10, and 11. We show here the comparison with Human, rat, shark, and amphioxus sequences. Using Teleost fish or coelacanth sequences instead of mammalian data yield qualitatively the same results (data not shown). 


\section{Results}

Only the paralog groups $1,2,3,4,8,9,10$, and 11 could be used for our purposes because (i) only a single short Hox-13 sequence from Lampetra planeri was found in the databanks, (ii) there does not seem to be a Hox-12 gene at all in lampreys, and (iii) the available sequences are too short and too conserved to distinguish unambigously between members of the paralog groups 5, 6, and 7, see also (Force et al., 2002; Irvine et al., 2002).

The comparison of mammalian, shark, lamprey, and amphioxus sequences for a given paralog group presents a striking pattern. We find that the lamprey sequences cluster together outside the gnathostome Hox sequences for paralog groups 11, 10, 9, 8, and 4 according to the split decomposition analysis, Fig. 2. Paralog group 1 is at least consistent with this picture. The single paralog group 3 sequence shows affinity with the shark HoxA sequence but is well separated from the mammalian HoxA-3 genes in the split data. The PmE2 sequence, which is physically linked to Pm3, is more similar to the mammalian HoxB-2 genes. Replacing the rat sequences by coelacanth sequences from the work of Koh et al. (2003) yields very similar results (data not shown).

The same picture is obtained from maximally parsimonious trees, see Table 2 in the Appendix, for groups 11, 10, 9, and 8. In contrast to the split decomposition method, the best trees for both paralog group 3 and 2 place the lamprey and amphioxus sequences together and as outgroup to the gnathostome clusters. Furthermore, the single Hox-13 sequence of Lampetra planeri reported by Sharman and W. (1998) branches outside the other vertebrate genes. Paralog group 1 yields one tree that shows the $1 \mathrm{w}$ sequence outside the mammalian cluster and two alternative trees placing $1 \mathrm{w}$ with mammalian $A$ clusters. In paralog group 4 the lamprey sequences also lie outside the mammalian clusters but form two separate branches. In no case do we find a clear assignment of the lamprey clusters to either a single or a pair of mammalian and/or fish clusters.

At present the genomic context of only a single lamprey Hox gene, Hox-W10a from Petromyzon marinus, has been published. Irvine et al. (2002) report footprint clusters shared with both HoxA and HoxC clusters. The footprint cliques detected by the tracker program in a comparison with Fugu, Shark, Human, and Ciona Hox clusters are summarized in Table 3 in the Appendix. Non-colinear cliques have been removed because they are most likely not homologous (Prohaska et al., 2003a). There is no clear evidence that the non-coding part of the Pm18 sequence is more closely related to either a particular single gnathostome cluster or pair of clusters. The total length of available footprints is unfortunately insufficient for an independent reconstruction of the phylogeny. The most significant footprint cliques are those shared with the HoxA and $H_{0 x C}$ clusters, in particular, and an element designated $\boldsymbol{p} \boldsymbol{p}$ that is most likely the proximal promotor of the Hox-10 genes and also appears in the HoxD clusters. The elements $\boldsymbol{A} \mathbf{1}, \boldsymbol{A} \boldsymbol{2}, \boldsymbol{C 1}$, and $\boldsymbol{C} 3$ are described already in the work of Irvine et al. (2002). Both $\boldsymbol{A} \boldsymbol{1}$, and $\boldsymbol{A} \mathscr{2}$ were also detected in comparisons of Hox $A$ clusters only by Chiu et al. (2002). It is interesting to note that both $\boldsymbol{A} \boldsymbol{2}$ and the $\boldsymbol{C} \boldsymbol{1}, \boldsymbol{C} \boldsymbol{2}$ motifs

\footnotetext{
${ }^{1}$ http://genome.jgi-psf.org/fugu6/fugu6.home.html
} 
also have their counterparts in the Human HoxB cluster, even though it lacks the $H o x B$-10 gene.

\section{Discussion}

The re-evaluation of the available lamprey hox genes strongly supports an independent origin of the three (or four) lamprey Hox clusters and suggest that the common ancestor of agnathans and gnathostomes had only a single Hox cluster. This is consistent with the $D l x$ gene phylogeny described by Neidert et al. (2001). These authors proposed that a tandem duplication of an ancestral $D l x$ gene predated the divergence of lampreys from gnathostomes, which was then followed by independent chromosomal or genome duplications and gene loss in each lineage. Our evaluation of the Hox clusters supports this hypothesis. Similar patterns have been reported for other developmentally important gene families. The neural crest marker AP-2, for which no duplicates have been found in lampreys, also fails to group with any one gnathostome AP-2 isoform (Meulemans and Bronner-Fraser, 2002). Consistent with an independent duplication history it is impossible to assign any one of the lamprey (and hagfish) Otx sequences to one of the three classes identified in gnathostomes (Germot et al., 2001).

The phylogenetic signal in the Hox clusters is not as strong as one would like so that a definitive result will have to await more complete sequencing. This will in particular allow the unambiguous identification of the genes of paralog group 5,6 , and 7, and their use as additional phylogenetic information. At present, at least, the publicly available sequence information does not contain evidence for a Hox-cluster duplication preceeding our common ancestor with the lampreys.

Acknowledgments. Funding for this research is gratefully acknowledged: DFG Bioinformatics Initiative BIZ-6/1-2 to SJP, CF, and PFS.

\section{References}

Amores A, Force A, Yan YL, Joly L, Amemiya C, Fritz A, Ho RK, Langeland J, Prince V, Wang YL, Westerfield M, Ekker M, Postlethwait JH, 1998. Zebrafish hox clusters and vertebrate genome evolution. Science 282:1711-1714.

Bandelt HJ, Dress AWM, 1992. A canonical decomposition theory for metrics on a finite set. Adv Math 92:47.

Carr JL, Shashikant CS, J. BW, Ruddle FH, 1998. Molecular evolution of Hox gene regulation: cloning and transgenic analysis of the lamprey HoxQ8 gene. J Exp Zool 280:73-85.

Chiu Ch, Amemiya C, Dewar K, Kim CB, Ruddle FH, Wagner GP, 2002. Molecular evolution of the HoxA cluster in the three major gnathostome lineages. Proc Natl Acad Sci USA 99:5492-5497.

Dehal P, Satou Y, Campbell RK, Chapman J, Degnan B, De Tomaso A, Davidson B, Di Gregorio A, Gelpke M, Goodstein DM, Harafuji N, Hastings KEM, Ho I, Hotta K, Huang W, Kawashima T, Lemaire P, Martinez D, Meinertzhagen IA, Necula S, Nonaka M, Putnam N, Rash S, Saiga H, Satake M, Terry A, Yamada L, Wang HG, 
Awazu S, Azumi K, Boore J, Branno M, Chin-bow S, DeSantis R, Doyle S, Francino P, Keys DN, Haga S, Hayashi H, Hino K, Imai KS, Inaba K, Kano S, Kobayashi K, Kobayashi M, Lee BI, Makabe KW, Manohar C, Matassi G, Medina M, Mochizuki Y, Mount S, Morishita T, Miura S, Nakayama A, Nishizaka S, Nomoto H, Ohta F, Oishi K, Rigoutsos I, Sano M, Sasaki A, Sasakura Y, Shoguchi E, Shin-i T, Spagnuolo A, Stainier D, Suzuki MM, Tassy O, Takatori N, Tokuoka M, Yagi K, Yoshizaki F, Wada S, Zhang C, Hyatt PD, Larimer F, Detter C, Doggett N, Glavina T, Hawkins T, Richardson P, Lucas S, Levine YKM, Satoh N, Rokhsar DS, 2002. The draft genome of Ciona intestinalis: Insights into chordate and vertebrate origins. Science 298:2157-2167.

Escriva H, Manzon L, Youson J, Laudet V, 2002. Analysis of lamprey and hagfish genes reveals a complex history of gene duplications during early vertebrate evolution. Mol Biol Evol 19:1440-1450.

Felsenstein J, 1989. Phylip - phylogeny inference package (version 3.2). Cladistics 5:164-166.

Force A, Amores A, Postlethwait JH, 2002. Hox cluster organization in the jawless vertebrate Petromyzon marinus. J Exp Zool Mol Dev Evol 294:30-46.

Garcia-Fernández J, Holland PW, 1994. Archetypal organization of the amphioxus hox gene cluster. Nature 370:563-566.

Germot A, Lecointre G, Plouhinec JL, Le Mentec C, Girardot F, Mazan S, 2001. Structural evolution of otx genes in craniates. Mol Biol Evol 18:1668-1678.

Holland PW, Garcia-Fernandez J, 1996. Hox genes and chordate evolution. Dev Biol 173:382-395.

Huson DH, 1998. Splitstree: analyzing and visualizing evolutionary data. Bioinformatics 14:68-73.

Irvine SQ, Carr JL, Bailey WJ, Kawasaki K, Shimizu N, Amemiya CT, Ruddle FH, 2002. Genomic analysis of Hox clusters in the sea lamprey, Petromyzon marinus. J Exp Zool Mol Dev Evol 294:47-62.

Koh EGL, Lam K, Christoffels A, Erdmann MV, Brenner S, Venkatesh B, 2003. Hox gene clusters in the indonesian coelacanth, Latimeria menadoensis. Proc Natl Acad Sci USA 100:1084-1088.

McGinnis W, Krumlauf R, 1992. Homeobox genes and axial patterning. Cell 68:283302.

Meulemans D, Bronner-Fraser M, 2002. Amphioxus and lamprey AP-2 genes: implications for neural crest evolution and migration patterns. Development 129:49534962.

Morgenstern B, 1999. DIALIGN 2: improvement of the segment-to-segment approach to multiple sequence alignment. Bioinformatics 15:211-218.

Neidert AH, Virupannavar V, Hooker GW, Langeland JA, 2001. Lamprey $d l x$ genes and early vertebrate evolution. Proc Natl Acad Sci USA 98:1665-1670.

Pendleton J, Nagai BK, Murtha MT, Ruddle FH, 1993. Expansion of the Hox gene family and the evolution of chordates. Proc Natl Acad Sci USA 90:6300-6304.

Prohaska S, Fried C, Flamm C, Wagner G, Stadler PF, 2003a. Surveying phylogenetic footprints in large gene clusters: Applications to Hox cluster duplications. J Mol Biol Submitted; SFI preprint \#03-02-011. 
Prohaska SJ, Fried C, Amemiya CT, Ruddle FH, Wagner GP, Stadler PF, 2003b. The shark HoxN cluster is homologous to the human HoxD cluster Submitted.

Schubert FR, Nieselt-Struwe K, Gruss P, 1993. The antennapedia-type homeobox genes have evolved from three precursors separated early in metazoan evolution. Proc Natl Acad Sci USA 90:143-147.

Semple C, Steel M, 2003. Phylogenetics. Oxford UK: Oxford University Press.

Sharman AC, W. HP, 1998. Estimation of Hox gene cluster number in lampreys. Int J Dev Biol 42:617-620.

Stellwag EJ, 1999. Hox gene duplications in fish. Cell Devel Biol 10:531-540. 


\section{Appendix (or Supplement)}

Table 1. Lamprey Hox sequences used in this study.

\begin{tabular}{|c|c|c|c|c|c|c|c|}
\hline \multirow[t]{3}{*}{ Hox } & \multicolumn{5}{|c|}{ Petromyzon marinus } & \multicolumn{2}{|c|}{ Lampetra planeri } \\
\hline & \multicolumn{3}{|c|}{ genomic clones } & \multicolumn{4}{|c|}{ PCR surveys } \\
\hline & Irvine & Force & Acc. No. & Pendleton & Acc. No. & Sharman & Acc. No. \\
\hline 13 & & & & & & Lp13A & AF044814 \\
\hline \multirow[t]{4}{*}{11} & Y11 & $11 \mathrm{w}$ & AF410923 & 11.1 & & & \\
\hline & Z11a & & AF410924 & 11.8 & & & \\
\hline & Z11b & & AF410925 & & & & \\
\hline & & & & 11.6 & & & \\
\hline \multirow[t]{3}{*}{10} & $\mathrm{X} 10$ & $\mathrm{Hx} 13(9)$ & AF410922 & $10 \mathrm{x}$ & L14900 & \multirow{3}{*}{ Lp10B } & \multirow{3}{*}{ AF044812 } \\
\hline & W10a & $10 \mathrm{w}$ & AF410920 & $10 \mathrm{w}$ & L14895 & & \\
\hline & W10b & & AF410921 & & & & \\
\hline \multirow[t]{4}{*}{9} & V9 & $9 \mathrm{y}$ & AF410919 & $\overline{9 v}$ & L14889 & Lp9A & AF044809 \\
\hline & & & & $9 \mathrm{~s}$ & L14911 & & \\
\hline & T9 & $9 \mathrm{w}$ & AF410918 & $9 \mathrm{t}$ & L14894 & Lp9B & AF044810 \\
\hline & & $9 \mathrm{x}$ & & $9 \mathrm{u}$ & L14910 & Lp9C & AF044811 \\
\hline \multirow[t]{3}{*}{8} & $\mathrm{R} 8$ & & AF035588 & $8 \mathrm{r}$ & & Lp8A & AF044807 \\
\hline & Q8 & & AF035591 & & & & \\
\hline & Q8a & & AF035589 & $8 q$ & L14901 & Lp8B & AF044808 \\
\hline \multirow[t]{5}{*}{4} & G4 & $4 y$ & AF410911 & $4 \mathrm{~g}$ & L14912 & & \\
\hline & & $4 \mathrm{w}$ & AF434666 & $4 n$ & L14896 & ? Lp4-7B & AF044803 \\
\hline & & $4 \mathrm{x}$ & AY056469 & 41 & L14891 & ? Lp4-7A & AF044802 \\
\hline & & & & & & ? Lp4-7E & AF044806 \\
\hline & & & & $(4 \mathrm{~h}$ & L14909) & & \\
\hline 3 & 3 & $3 y$ & AF410909 & & & Lp3A & AF044801 \\
\hline 2 & E2 & & AF410908 & $2 \mathrm{e}$ & & Lp2A & AF044800 \\
\hline \multirow[t]{4}{*}{1} & $1 \mathrm{~B}$ & $1 \mathrm{w}$ & AF434665 & $1 \mathrm{~b}$ & L14902 & Lp1B & AF044798 \\
\hline & & & & $1 \mathrm{a}$ & L14893 & Lp1A & AF044797 \\
\hline & & & & $1 \mathrm{c}$ & L14908 & Lp1C & AF044799 \\
\hline & & & & (1d & L14904) & & \\
\hline
\end{tabular}

The sequence shown in parentheses are not included because we could not confirm their assignment to a paralog group based on their nucleic acid sequence. 
Table 2. Maximum parsimony trees of the homeobox sequences obtained with the program dnapenny from the phylip package Felsenstein (1989). Petromyzon marinus, Pm, sequences are indicated in bold. For Hox-13 we used the short Lampetra planeri, Lp sequence. Grey boxes indicated that all available lamprey paralogs form a subtree, dark gray boxes are used when all lamprey and the amphioxus sequence are separated from the vertebrate hox clusters. Horizontal lines indicate the two exons of the Hox-10 in the HoxA, HoxB, and $\operatorname{Hox} D$, as well as the Ciona Hox cluster Dehal et al. (2002).

\begin{tabular}{|c|c|}
\hline Hox & Maximum parsimony tree \\
\hline \multirow[t]{2}{*}{13} & 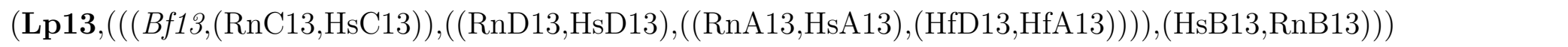 \\
\hline & $(\mathbf{L p 1 3},(((B f 13,(\mathrm{RnC13}, \mathrm{HsC13})),((\mathrm{RnD} 13, \mathrm{HsD} 13),(\mathrm{HfD} 13,((\mathrm{RnA13}, \mathrm{HsA13}), \mathrm{HfA13})))),(\mathrm{HsB13}, \mathrm{RnB13})))$ \\
\hline 11 & $(($ Bf11,(PmY11,(PmZ11a,PmZ11b) $)),(((H f 11 D,(H s 11 C, R n 11 C)),(($ Rn11A,Hs11A $), H f 11 A)),($ Rn11D,Hs11D $)))$ \\
\hline 10 & $(($ Bf10,(PmX10,(PmW10b,PmW10a) $)),(($ Rn10A,Hs10A $),(H f 10 A,((R n 10 C, H s 10 C),((H s 10 D, R n 10 D), H f 10 D)))))$ \\
\hline \multirow[t]{2}{*}{9} & ( (BfHox9,(HoxT9,HoxV9)),(((Hs9D,Rn9D),((((Rn9A,Hs9A),Hf9A),(Hs9B,Rn9B)),((Hs9C,Rn9C),Hf9D))))) \\
\hline & ( (BfHox9,(HoxT9,HoxV9)),$(((((H s 9 D, R n 9 D),((H s 9 C, R n 9 C), H f 9 D)),(H f 9 A,(H s 9 B, R n 9 B))),($ Rn9A,Hs9A $))))$ \\
\hline 8 & $\left((\right.$ Bf8,(PmQ8,(PmR8,PmQ8a)) $\left.),\left(\left(\mathrm{HsC}_{\mathbf{H}},(\mathrm{HsB} 8, \mathrm{Rn} 8 \mathrm{~B})\right),(\mathrm{HfD} 8, \mathrm{HsD} 8)\right)\right)$ \\
\hline \multirow[t]{2}{*}{4} & $((B f 4, \mathbf{P m G} 4),(((\mathbf{P m} 4 \mathbf{x}, \mathbf{P m} 4 \mathrm{w}),(\mathrm{Hs} 4 \mathrm{~A},(\mathrm{Hf} 4 \mathrm{~A},(\mathrm{Rn} 4 \mathrm{D}, \mathrm{Hs} 4 \mathrm{D})))),(\mathrm{Hs} 4 \mathrm{~B}, \mathrm{Rn} 4 \mathrm{~B})),(\mathrm{Hs} 4 \mathrm{C}, \mathrm{Rn} 4 \mathrm{C}))$ \\
\hline & $((B f 4, \mathbf{P m G} 4),((\mathbf{P m} 4 \mathbf{x}, \mathbf{P m} 4 \mathbf{w}),((\mathrm{Hs} 4 \mathrm{~B}, \mathrm{Rn} 4 \mathrm{~B}),(\mathrm{Hs} 4 \mathrm{~A},(\mathrm{Hf} 4 \mathrm{~A},(\mathrm{Rn} 4 \mathrm{D}, \mathrm{Hs} 4 \mathrm{D}))))),(\mathrm{Hs} 4 \mathrm{C}, \mathrm{Rn} 4 \mathrm{C}))$ \\
\hline 3 & $(($ Bf3,Pm3),$(\mathrm{Hf} 3 \mathrm{~A},(\mathrm{Hs} 3 \mathrm{D},((\mathrm{Hs} 3 \mathrm{~A}, \mathrm{Rn} 3 \mathrm{~A}),(\mathrm{Hs} 3 \mathrm{~B}, \mathrm{Rn} 3 \mathrm{~B})))))$ \\
\hline \multirow[t]{2}{*}{2} & $(($ Bf2,PmE2),$(((\mathrm{Hf} 2 \mathrm{~A},(\mathrm{Rn} 2 \mathrm{~A}, \mathrm{Hs} 2 \mathrm{~A})),(\mathrm{Rn} 2 \mathrm{~B}, \mathrm{Hs} 2 \mathrm{~B}))))$ \\
\hline & $(($ Bf2,PmE2),$((((\mathrm{Hf} 2 \mathrm{~A},(\mathrm{Rn} 2 \mathrm{~A}, \mathrm{Hs} 2 \mathrm{~A})), \mathrm{Hs} 2 \mathrm{~B}), \mathrm{Rn} 2 \mathrm{~B})))$ \\
\hline \multirow{3}{*}{1} & $((B f 1, \mathbf{P m} 1 \mathbf{w}),((((\mathrm{Hs} 1 \mathrm{D}, \mathrm{Rn} 1 \mathrm{D}), \mathrm{Hf1A}),(\mathrm{Rn} 1 \mathrm{~A}, \mathrm{Hs} 1 \mathrm{~A})),(\mathrm{Hs} 1 \mathrm{~B}, \mathrm{Rn} 1 \mathrm{~B})))$ \\
\hline & $(B f 1,(((H s 1 D, R n 1 D), H f 1 A),((H s 1 B, R n 1 B),((R n 1 A, H s 1 A), P m 1 w))))$ \\
\hline & $(B f 1,(((\mathrm{Hs} 1 \mathrm{D}, \mathrm{Rn} 1 \mathrm{D}),(\mathrm{Hs} 1 \mathrm{~B}, \mathrm{Rn} 1 \mathrm{~B})),(\mathrm{Hf} 1 \mathrm{~A},((\mathrm{Rn} 1 \mathrm{~A}, \mathrm{Hs} 1 \mathrm{~A}), \mathbf{P m} 1 \mathbf{w}))))$ \\
\hline
\end{tabular}


Table 3. Summary of co-linear footprint cliques produced by the tracker program in the range of the Petromyzon marinus Pm18 sequence. Hs Homo Sapiens, Hf Heterodontus fransisci, Tr Takifugu rubripes, Ci Ciona intestinalis. Numbers in parentheses are non-colinear with the footprints in this species. The last column marks previously described footprints. $\boldsymbol{p} \boldsymbol{p}$ is the proximal promotor of the Hox-10 gene, numbers in sans serif font are cliques listed in Prohaska et al. (2003a) for a comparison of HoxA clusters. PFC, "phylogenetic footprint cluster", names from Chiu et al. (2002) are given in normal text font.

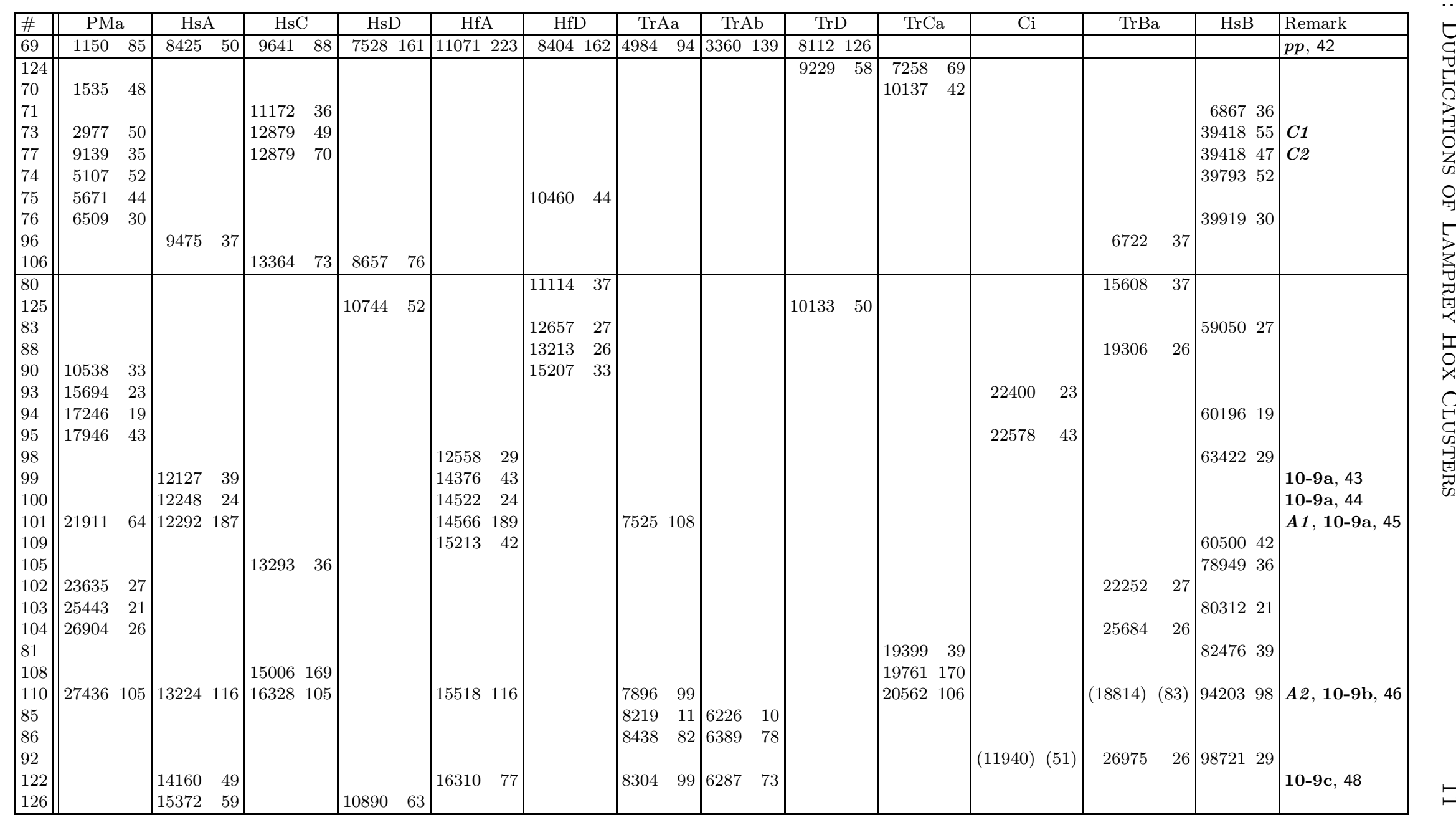

